\title{
Co-Culture with Bifidobacterium catenulatum Improves the Growth, Gut Colonization, and Butyrate Production of Faecalibacterium prausnitzii: In Vitro and In Vivo Studies
}

\author{
Heejung Kim ${ }^{1}{ }^{1}$, Yunju Jeong ${ }^{1}$, Sini Kang ${ }^{1}$, Hyun Ju You ${ }^{2, *}$ and Geun Eog Ji ${ }^{1,3, *}$ \\ 1 Department of Food and Nutrition, Research Institute of Ecology, Seoul National University, \\ Seoul 08826, Korea; bonobono007@snu.ac.kr (H.K.); tanklov0@snu.ac.kr (Y.J.); kangsini@snu.ac.kr (S.K.) \\ 2 Institute of Health and Environment, Graduate School of Public Health, Seoul National University, \\ Seoul 08826, Korea \\ 3 Research Center, BIFIDO Co., Ltd., Hongcheon 25117, Korea \\ * Correspondence: dhlover1@snu.ac.kr (H.J.Y.); geji@snu.ac.kr (G.E.J.); Tel.: +82-2-880-2790 (H.J.Y.); \\ $+82-2-880-6282$ (G.E.J.)
}

Received: 6 May 2020; Accepted: 23 May 2020; Published: 25 May 2020

\begin{abstract}
Faecalibacterium prausnitzii is a major commensal bacterium in the human gut. It produces short-chain fatty acids that promote intestinal health. However, the bacterium is extremely oxygen-sensitive, making it difficult to develop as a probiotic. To facilitate practical application of F. prausnitzii, we investigated factors that affect its growth and mammalian gut colonization. We evaluated cross-feeding interactions between F. prausnitzii and seven Bifidobacterium strains, and the anti-inflammatory properties of bacterial metabolites produced in co-culture, in vitro and in vivo. Co-culture of $F$. prausnitzii and Bifidobacterium catenulatum, with fructooligosaccharides as an energy source, resulted in the greatest viable cell-count and butyrate production increases. Further, the co-culture supernatant reduced the amount of proinflammatory cytokines produced by HT-29 cells and RAW 264.7 macrophages, an effect that was similar to that of butyrate. Furthermore, feeding mice both Faecalibacterium and Bifidobacterium enhanced F. prausnitzii gut colonization. Finally, feeding the co-culture supernatant decreased interleukin 8 levels in the colon and increased butyrate levels in the cecum in the dextran sodium sulfate-induced colitis mouse model. These observations indicate that the Faecalibacterium-Bifidobacterium co-culture exerts an anti-inflammatory effect by promoting F. prausnitzii survival and short-chain fatty acid production, with possible implications for the treatment of inflammatory bowel disease.
\end{abstract}

Keywords: Faecalibacterium prausnitzii; Bifidobacterium catenulatum; cross-feeding; anti-inflammation; butyrate

\section{Introduction}

Inflammatory bowel disease (IBD), including Crohn's disease and ulcerative colitis, is characterized by inflammation and tissue damage in the distal ileum and colon. IBD causes chronic intestinal inflammation and is diagnosed in adolescents and children. The incidence of IBD in the wealthy western countries has been increasing since the mid-twentieth century. The incidence of IBD in Asia in the past 20 years has also increased, and IBD is currently recognized as an emerging global challenge [1-4].

The pathogenesis of IBD is driven by genetic predisposition and environmental factors, such as westernized diet and socioeconomic development. Gut dysbiosis is considered a novel pivotal factor associated with IBD pathogenesis and is characterized by a decreased diversity of the gut microbiota. Recent advances in next-generation sequencing allowed insight into the alteration of the gut microbiota, its composition, and physiological function in IBD [5]. Anti-inflammatory drugs used for treating IBD 
partially relieve the disease symptoms; however, the use of the drugs results in side effects such as headache and diarrhea, which lead to a poor quality of life [6]. This calls for novel treatment strategies, such as the use of probiotics.

Faecalibacterium prausnitzii is a member of Clostridium cluster IV within the Firmicutes phylum and is one of the most abundant commensal bacteria in the gut of healthy adults [7]. According to recent studies, F. prausnitzii exerts anti-inflammatory effect in vitro and in vivo. For instance, it strongly induces interleukin (IL)-10 production by the human peripheral blood mononuclear cells, and its culture supernatant reduces IL- 8 secretion by HT-29 cells induced by tumor necrosis factor (TNF)- $\alpha$ [8,9]. In addition, administration of F. prausnitzii and its culture supernatant has a protective effect in a mouse model of dinitrobenzene sulfonic acid induced chronic colitis and dextran sodium sulfate (DSS)-induced colitis [10-13]. Importantly, the abundance of F. prausnitzii is reduced in individuals with IBD. Therefore F. prausnitzii serves as a potential biomarker for the diagnosis and prognosis of gut diseases [14,15].

F. prausnitzii produces butyrate by using butyrate kinase or butyryl CoA:acetate CoA transferase route, which exerts an anti-inflammatory effect by inhibiting the differentiation of Thelper 17 cells [16,17]. Further, butyrate is a key energy source for the colonocyte and acts as an important regulator of gene expression, differentiation, and apoptosis of the host cell [18]. Butyrate and other short-chain fatty acids (SCFA) are also produced during fermentation of the dietary fiber in the colon. Diet-in particular, the type and quantity of substrates that survive the journey through the upper digestive system to the gut-affects the concentrations and relative proportions of SCFA in the gut $[19,20]$.

Dietary fiber, such as resistant starch, inulin, and fructooligosaccharides (FOS), are major sources of energy for colonic bacteria [21]. The yield of bacterial metabolites from fermentable carbohydrates depends on the composition of the microbial community and the metabolic properties of the individual bacteria [22]. Cross-feeding bacteria use metabolites generated by fermentation by other bacteria as an energy source. For example, cross-feeding between Bifidobacterium and Faecalibacterium has been demonstrated in co-cultures in vitro. Bifidobacterium is the most abundant bacterial colonizer of the neonatal gut and is beneficial to the host intestinal health because of its metabolizing capacity to metabolize human milk oligosaccharides and exert immuno-modulatory functions in early age. Bifidobacteria produce lactate and acetate, but not butyrate [23]. In contrast, F. prausnitzii is an acetate-consuming and butyrate-producing bacterium and employs the butyryl CoA:acetate CoA transferase route for butyrate production [24]. It uses acetate produced by bifidobacteria in a starch- or FOS-containing medium, and this enhances its growth and butyrate production [25].

Despite the importance of F. prausnitzii as a next-generation probiotic candidate, to date, few studies using isolated strains have been conducted, with few functional approaches $[7,11,13,26]$. That is because this bacterium is difficult to cultivate even under anaerobic conditions. F. prausnitzii is an extremely oxygen-sensitive bacterium. It is particularly sensitive to exposure to air, surviving under such conditions for less than $2 \mathrm{~min}$ [25]. Therefore, intestinal delivery of F. prausnitzii remains challenging. Several approaches to increase the intestinal colonization and survival of F. prausnitzii have been attempted. Some methods for colonizing F. prausnitzii in the intestinal mucosa can be found in relation to other bacteria. For example, recent studies in adult gnotobiotic rodents revealed that F. prausnitzii colonizes the gut only with an a priori presence of Bacteroides thetaiotaomicron or Escherichia coli [26]. On the other hand, F. prausnitzii can grow in the presence of oxygen, provided that the growth medium contains flavins and cysteine or glutathione as an electron acceptor [27]. The interaction between bifidobacteria and bacteria from the F. prausnitzii has not yet been tested in vivo.

In the current study, we aimed to evaluate the effect of $F$. prausnitzii and bifidobacterial co-culture on the promotion of F. prausnitzii growth within the mammalian intestinal environment and butyrate production. We showed that acetate and degraded dietary fiber produced by different Bifidobacterium species promote the growth of F. prausnitzii, with cross-feeding also resulting in increased butyrate production. In line with the immunomodulatory properties of butyrate, the co-culture supernatant of F. prausnitzii and Bifidobacterium catenulatum exerted anti-inflammatory effects in macrophages and 
a DSS-induced colitis mouse model. In addition, administration of B. catenulatum or the co-culture supernatant to mouse enhanced gut colonization of $F$. prausnitzii in vivo as assessed by $16 \mathrm{~S}$ rRNA sequencing and real-time quantitative PCR. These findings indicate that the co-culture of B. catenulatum and F. prausnitzii in fortified FOS media can be a useful strategy to enhance the growth and colonization of F. prausnitzii, increase butyrate production, and alleviate the symptoms of DSS-induced colitis.

\section{Materials and Methods}

\subsection{Bacterial Strains and Culture}

The fecal isolates F. prausnitzii ATCC 27768 and A2-165 and seven Bifidobacterium strains were used. Commercial strains of F. prausnitzii ATCC 27768, F. prausnitzii A2-165, and B. catenulatum KCTC 3221 were purchased from American Type Culture Collection (ATCC, Manassas, VA, USA), DSMZ-German Collection of Microorganisms and Cell Cultures (DSMZ, Braunschweig, Germany), and Korean Collection for Type Cultures (KCTC, Daejeon, Korea), respectively. Six Bifidobacterium strains were isolated from fecal samples of healthy infants in Korea and identified by 16rRNA gene sequencing. F. prausnitzii ATCC 27768 and F. prausnitzii A2-165 were cultured anaerobically for $24 \mathrm{~h}$ at $37^{\circ} \mathrm{C}$ in modified reinforced clostridial broth in Hungate tubes. B. catenulatum KCTC 3221, B. bifidum BGN4, B. $\log u m$ RD72, B. animalis ATCC 25557, B. animalis subsp. lactis RD68, B. animalis subsp. lactis SH5, and B. animalis subsp. lactis ad011 were incubated anaerobically in de Man, Rogosa, and Sharpe (MRS) medium (Difco, Detroit, MI, USA) supplemented with $0.05 \%(w / v)$ L-cysteine for $18 \mathrm{~h}$ at $37^{\circ} \mathrm{C}$.

\subsection{Assessment of Carbohydrate Utilization and Bacterial Growth}

Co-culture experiments with selected strains, and in the presence of specific carbon sources, were performed to study the interaction between F. prausnitzii ATCC 27768 and Bifidobacterium species. For each strain, overnight culture was inoculated to $1 \%(v / v)$ in $10 \mathrm{~mL}$ Yeast extract, Casitone, FOS (YCFOS) medium [25]. The co-cultures were set up by mixing the different bacteria in a 1:1 ratio. Yeast extract, casitone, and fatty acid (YCFA) broth without SCFA was supplemented with glucose, starch, inulin, or FOS (3.5 g/L for each carbohydrate). All manipulations were performed in an anaerobic chamber (Coy Lab, Grass Lake, MI, USA) at $37^{\circ} \mathrm{C}$ under an atmosphere of $80 \% \mathrm{~N}_{2}, 10 \% \mathrm{H}_{2}$, and $10 \% \mathrm{CO}_{2}$. Liquid medium in Hungate tube was placed in an anaerobic chamber immediately after autoclaving. LYBHI (brain-heart infusion medium supplemented with $0.5 \%$ yeast extract) (Difco, Detroit, MI, USA) agar plates were prepared and equilibrated in an anaerobic chamber for $24 \mathrm{~h}$ before use. Bacterial growth in monoculture and co-culture was monitored by measuring culture optical density $\left(\mathrm{OD}_{650 \mathrm{~nm}}\right.$ ), viable cell counts, $\mathrm{pH}$, and SCFA production (Sections 2.2.1 and 2.2.2). For Opitical density (OD) measurements and SCFA analysis, the cultures were sampled after 0, 9, and $24 \mathrm{~h}$ of incubation. The bacterial supernatants used in in vitro cell culture experiments were collected after cultivation for $24 \mathrm{hr}$ of Faecalibacterium and Bifidobacterium, respectively. The samples were centrifuged, and the medium was immediately stored at $-80{ }^{\circ} \mathrm{C}$ until use.

\subsection{1. $\mathrm{pH}$ Measurements and Viable Cell Counts}

All procedures were performed in an anaerobic chamber. Monocultures and F. prausnitzii ATCC 27768 co-cultures with B. catenulatum KCTC 3221 or B. animalis ATCC 25527 were incubated in YCFOS medium without SCFA or plated on LYBHI medium at $37{ }^{\circ} \mathrm{C}$ [28]. The initial medium $\mathrm{pH}$ was determined by using a pH meter (model K2000-pH; Istek, Inc., Seoul, Korea). The pH was measured after $0,7,18$, and $24 \mathrm{~h}$ of incubation. For viable cell counts, cells from the monocultures and co-cultures were plated on LYBHI agar medium and incubated at $37^{\circ} \mathrm{C}$. The resultant colonies were counted after 2 d. F. prausnitzii forms transparent colonies, whereas bifidobacteria form yellow colonies. The data are expressed as $\log 10[$ colony-forming units $(\mathrm{CFU})] / \mathrm{mL}$. 


\subsubsection{SCFA Determinations}

SCFA levels were determined as previously described [29]. Briefly, organic acids, such as acetate, lactate, formate, and butyrate, were analyzed by high-performance liquid chromatography. A YL9100 HPLC system (Young-lin, Anyang, Korea), equipped with an RI detector and Aminex HPX-87H column (300 × $7.8 \mathrm{~mm}$; Bio-Rad Laboratories, Hercules, CA, USA), was used. Cecum samples $(100 \mathrm{mg})$ were homogenized in phosphate-buffered saline $(400 \mu \mathrm{L})$. Then, $25 \%(\mathrm{w} / \mathrm{v})$ meta-phosphoric acid (Sigma-Aldrich, St. Louis, MO, USA) was added to the homogenate at a 1:5 ratio, and the samples were incubated at room temperature for $30 \mathrm{~min}$. The samples were centrifuged at $15,000 \times g$ for $15 \mathrm{~min}$, and the supernatants were stored at $-80^{\circ} \mathrm{C}$ until further processing [30]. The liquid culture medium was centrifuged at $18,000 \times g$ for $15 \mathrm{~min}$, and the supernatant was then filtered through a $0.2 \mu \mathrm{m}$ filter. For the analysis, $0.005 \mathrm{M}$ sulfuric acid (J.T. Baker, Phillipsburg, MA, USA) was the mobile phase, with a flow rate of $0.6 \mathrm{~mL} / \mathrm{min}$. The concentration of each organic acid was normalized to the corresponding concentrations of external SCFA standards and is expressed in $\mathrm{mM}$.

\subsection{Cell Culture and Determination of Immunomodulatory Properties of Culture Supernatants}

HT-29 cells and RAW 264.7 macrophages were obtained from Korea Cell Line Bank (KCLB, Daejeon, Korea). HT-29 cells $\left(1 \times 10^{5}\right.$ cells/well $)$ and RAW 264.7 macrophages $\left(1 \times 10^{5}\right.$ cells/well $)$ were seeded into 24-well plates in Dulbecco's modified Eagle's medium supplemented with 10\% heat-inactivated fetal bovine serum (Welgene Inc., Daegu, Korea) and $1 \%$ antibiotic antimycotic solution (Sigma-Aldrich, St. Louis, MO, USA) and cultured for $3-4 \mathrm{~d}$ at $37{ }^{\circ} \mathrm{C}$ under $5 \% \mathrm{CO}_{2}$. Then, the cells were stimulated with $0.1 \mu \mathrm{g} / \mathrm{mL}$ lipopolysaccharide (LPS) (Sigma-Aldrich St. Louis, MO, USA). Next, 10\% (v/v) of bacterial supernatant (pH 7.0) was added in Dulbecco's modified Eagle's medium in a total volume of $1 \mathrm{~mL}$. The plates were incubated for $6 \mathrm{~h}$ at $37^{\circ} \mathrm{C}$ under $5 \% \mathrm{CO}_{2}$. All samples were analyzed in triplicate. After the incubation, cell culture supernatants were collected and stored at $-80^{\circ} \mathrm{C}$ until further analysis. IL-6, TNF- $\alpha$, and IL-8 levels were determined by using enzyme-linked immunosorbent assay kits (BD Biosciences, San Jose, CA, USA) according to the manufacturer's instructions [8].

\subsection{Animals and Experimental Design}

The experiments were approved by the ethics committee of Seoul National University (Institutional Animal Care and Use Committee, experiment 1 approval number, approval date: SNU-181127-11-1, 2019-03-25; experiment 2 approval number, approval date: SNU-191120-3, 2019-11-20). The mice were maintained at the animal facility of Seoul National University. Two different experiments were performed. In experiment 1, 24 male C57BL/6 mice (5-6-week-old, 18-21 g; OrientBio, Sungnam, Korea) were used. Mice were acclimatized for 1 week prior to the experiment. The animals were separated into four groups ( $n=6$ per group, $n=2$ per cage): mice administered with live F. prausnitzii (F group), mice administered with live F. prausnitzii and B. catenulatum (fed both bacteria (FB) group), mice administered with live B. catenulatum (B group), and mice administered with sterile saline (C group). Live bacteria were administered in $200 \mu \mathrm{L}$ doses (see Section 2.4.1), daily, by gavage, for $14 \mathrm{~d}$. All mouse feces were collected before sacrifice $(n=6$, respectively), and fecal bacterial abundance was determined, as described in Sections 2.4.3 and 2.4.4. In experiment 2, 45 female C57BL/6 mice (8-week-old, 18-21 g, OrientBio, Sungnam, Korea) were used. The animals were separated into five groups ( $n=7-9$ per group, $n=2-3$ per cage): untreated mice ( $n$ group), mice that were administered DSS and YCFOS medium (M group), mice that were administered DSS and supernatant from F. prausnitzii and B. catenulatum co-culture (FB group), mice that were administered DSS and supernatant from F. prausnitzii culture (F group), and mice that were administered DSS and supernatant from B. catenulatum culture (B group). The groups with DSS-induced colitis received $2 \%$ $(w / v)$ DSS (molecular mass, 36,000-50,000 kDa; MP Biomedicals, Santa Ana, CA, USA) in drinking water, ad libitum, for $7 \mathrm{~d}$, followed by $2 \mathrm{~d}$ of plain tap water. The mice were given bacterial supernatant by gavage (see Section 2.4.1), and bacterial supernatants were administered in $200 \mu \mathrm{L}$ doses and 
were weighed daily. The animals were sacrificed on day 9, and organs were harvested for analysis. Six samples per group were processed for histopathology and reverse-transcription quantitative polymerase chain reaction (RT-qPCR) (Section 2.4.2).

\subsubsection{Sample Preparation for Administration}

For experiment 1, F. prausnitzii ATCC 27768 was cultured in a modified reinforced clostridial broth for $24 \mathrm{~h}$ at $37^{\circ} \mathrm{C}$. B. catenulatum KCTC 3221 was grown in MRS broth with L-cysteine for $18 \mathrm{~h}$ at $37^{\circ} \mathrm{C}$. The bacteria were harvested by centrifugation at $8000 \times g$ for $10 \mathrm{~min}$ at $4{ }^{\circ} \mathrm{C}$, washed in phosphate-buffered saline, and re-suspended in sterile saline. The cells were administered orally $\left(1 \times 10^{9} \mathrm{CFU}\right)$ in $200 \mu \mathrm{L}$ sterile saline. F. prausnitzii $\left(1 \times 10^{9} / 100 \mu \mathrm{L}\right)$ and B. catenulatum $\left(1 \times 10^{9} / 100 \mu \mathrm{L}\right)$ were administered together to the FB group. For experiment 2, F. prausnitzii A2-165 and B. catenulatum KCTC 3221 were grown in YCFOS medium at $37^{\circ} \mathrm{C}$ for $24 \mathrm{~h}$. Supernatant was collected from $10^{7}-10^{8} \mathrm{CFU} / \mathrm{mL}$ culture by centrifugation. The supernatant was lyophilized and stored at $-80^{\circ} \mathrm{C}$. The lyophilizate was reconstituted in a 20-times concentrated volume.

\subsubsection{DNA Extraction and Real-Time qPCR Amplification}

Total genomic DNA from the stool and cecum were extracted and purified by using a QIAamp Fast DNA Stool Mini kit (Qiagen, Germantown, MD, USA) according to the manufacturer's instructions, followed by bead beating using a TissueLyser system (Qiagen, Germantown, MD, USA). Bacterial genomic DNA was quantified using a Qubit3.0 fluorometer (Thermo Fisher Scientific, Waltham, MA, USA) in conjunction with the Qubit dsDNA High Sensitivity (HS) Assay Kit. DNA purified from each sample was used for RT-qPCR and 16S rRNA gene sequencing (Section 2.4.4). The abundance of Faecalibacterium and Bifidobacterium among the fecal microbiota was quantified by RT-qPCR, using the SYBR $^{\circledR}$ Premix Ex Taq ${ }^{\mathrm{TM}}$ and StepOne ${ }^{\mathrm{TM}}$ Real-Time PCR System. Faecalibacterium and Bifidobacterium abundances were quantified using the following primers: Frau223F, 5' -GAT GGC CTC GCG TCC GAT TAG-3' ${ }^{\prime}$, and Frau420R, 5' -CCG AAG ACC TTC TTC CTC C-3' [31]; Bif164F, 5' -GGG TGG TAA TGC CGG ATG-3', and Bif601R, 5'-TAA GCC ATG GAC TTT CAC ACC-3' [32], respectively. The PCR reaction was performed as follows: $95^{\circ} \mathrm{C}$ for $30 \mathrm{~s}$, followed by 40 cycles at $95{ }^{\circ} \mathrm{C}$ for $30 \mathrm{~s}, 95^{\circ} \mathrm{C}$ for $5 \mathrm{~s}$, and $60^{\circ} \mathrm{C}$ for $34 \mathrm{~s}$. The relative abundance of Faecalibacterium and Bifidobacterium was calculated using the cycle threshold method.

\subsection{3. $16 \mathrm{~S}$ rRNA Gene Amplification and Sequence Analysis}

Bacterial genomic DNA isolated from feces was analyzed by next-generation sequencing as previously described [29]. 16S rRNA gene amplification and index PCR were performed following the Illumina 16S Metagenomic Sequencing Library preparation guide (Illumina, San Diego, CA, USA). The V3-V4 region of the bacterial 16S rRNA gene was PCR-amplified using the primers 16S Amplicon PCR Forward primer, 5'-TCG TCG GCA GCG TCA GAT GTG TAT AAG AGA CAG CCT ACG GGN GGC WGC AG-3', and 16S Amplicon PCR Reverse primer, 5'-GTC TCG TGG GCT CGG AGA TGT GTA TAA GAG ACA GGA CTA CHV GGG TAT CTA ATC C-3'. The amplicons were attached to specific barcode sequences to compile the pooled library using a Nextera XT index kit (Illumina San Diego, CA, USA). The PCR products were purified using AMPure XP beads (Beckman Coulter, Pasadena, CA, USA). Diluted DNA (4 $\mathrm{nM})$ was pooled into a library. The pooled library was denatured with $1 \mathrm{~N} \mathrm{NaOH}$ and diluted to $9 \mathrm{pM}$ in HT1 hybridization buffer (Illumina San Diego, CA, USA). A PhiX control library (Illumina San Diego, CA) was also denatured and diluted. The pooled library was mixed with the PhiX control $(30 \%, v / v)$ and loaded onto a MiSeq ${ }^{\circledR}$ version 2 (500 cycle) reagent cartridge (Illumina San Diego, CA, USA) for sequencing. Paired-end FASTQ files were quality-filtered, and data were processed using Quantitative Insights Into Microbial Ecology 2 (QIIME2) (version 2019.7, https://qiime2.org). Alpha- and beta-diversity analysis and operational taxonomic unit assignment were done using the q2-diversity plugin within QIIME2. Linear discriminant analysis (LDA) effect size analysis (LEfSe) was then performed. 


\subsubsection{Histological Analysis and RT-qPCR of the Colon Tissue}

For RT-qPCR, total RNA was isolated from a $30 \mathrm{mg}$ sample of colon (stabilized in RNAlater) using an RNeasy mini kit (Qiagen, Germantown, MD, USA). RNA concentration was determined by NanoDrop. The first-strand complementary DNA was synthesized using a reverse-transcription kit (Invitrogen, Carlsbad, CA, USA). The complementary DNA was amplified using SYBR ${ }^{\circledR}$ Premix Ex Taq ${ }^{\mathrm{TM}}$ (Takara, Dalian, China). PCR was performed using the StepOne ${ }^{\mathrm{TM}}$ Real-Time PCR System (Applied Biosystems, Foster City, CA). The following qPCR primers were used: for Il8, IL-8 Forward, 5'-CTA GGC ATC TTC GTC CGT CC-3' , and IL-8 Reverse, 5' -TTC ACC CAT GGA GCA TCA GG-3' [33]; for 116 , IL-6 Forward, 5' ${ }^{\prime}$-CTG CAA GAG ACT TCC ATC CAG-3' ${ }^{\prime}$, and IL-6 Reverse, 5' GAC AGG TCT GTT GG-3' [34]; for Tnfa, TNF-a Forward, $5^{\prime}$-CAT CTT CTC AAA ATT CGA GTG ACA A-3', and TNF-a Reverse, 5'-TGG GAG TAG ACA AGG TAC AAC CC-3' [35]; and for Gapdh, GAPDH Forward, $5^{\prime}$-AGG TCG GTG AAC GGA TTT G-3' , and GAPDH Reverse, $5^{\prime}$-TGT AGA CCA TGT AGT TGA GGT CA-3' [36], respectively. Relative mRNA levels were determined using the comparative cycle threshold method. Gapdh was used as the reference gene.

For the histological analysis, the colon was fixed in $10 \%$ buffered formalin. The tissue was then dehydrated and embedded in paraffin. Then, $5 \mathrm{~mm}$ sections were prepared and stained with hematoxylin and eosin (H\&E). The severity of colitis in each section was semi-quantified using a scoring system of 0 to 4: 0 , normal crypt architecture; 1, crypt shortening, loss of the basal one-third portion of the crypt, with the lamina propria prominent and minimal inflammatory changes; 2 , crypt shortening, loss of the basal two-thirds portion of the crypt and thinning of the epithelium with mild inflammatory changes; 3 , loss of the entire crypt, with an intact epithelial layer and moderate inflammatory changes; and 4 , loss of the entire crypt and epithelial layer (erosion), with severe inflammatory changes.

\subsection{Statistical Analyses}

All statistical analyses were performed using GraphPad Prism version 8.0 (GraphPad, La Jolla, CA, USA). All graphics were prepared using the same program. The Kruskal-Wallis test and a non-parametric one-way analysis of variance were used for differential relative abundance analysis. Parametric analysis of variance was used for statistical analysis of several groups. All data are presented as the mean \pm SEM; $p<0.05$ was considered to indicate statistical significance.

\section{Results}

3.1. Effects of Mono- and Co-Culture, and Different Carbon Sources on Bacterial Growth, SCFA Production, Culture $\mathrm{pH}$, and Viable Cell Counts

\subsubsection{Growth of Bacteria in Mono- and Co-Cultures in the Presence of Different Carbon Sources}

In the current study, we aimed to identify the factors that affect $F$. prausnitzii growth in order to enable development of this bacterium as a probiotic. We first investigated the growth $\left(\mathrm{OD}_{650}\right)$ of F. prausnitzii ATCC 27768 and different Bifidobacteria in monoculture and co-culture, in the presence of different carbon sources (Figure 1). F. prausnitzii did not grow well on the carbon sources tested in monoculture. By contrast, Bifidobacterium growth in monoculture was carbon-source-dependent. Further, the stimulation of general bacterial growth in co-culture was also carbon-source-dependent. All bacteria grew best on glucose, with second-best growth on FOS. This indicated that the bacteria grew better in the presence of a short-chain sugar substrate (FOS) or monosaccharides (glucose) than in the presence of long-chain sugars, such as starch and inulin. After $24 \mathrm{~h}$ co-culture, the relative growth on FOS was $54.1-109 \%$ that of growth on glucose (23.1-62.3\% for inulin and $22-29.9 \%$ for starch). Compared with the growth on glucose, F. prausnitzii and B. catenulatum co-culture showed good growth on inulin $(62.3 \%)$ and FOS (109\%). In addition, B. catenulatum grew better on inulin and FOS than the other Bifidobacterium species tested. Co-culture on FOS resulted in better growth than co-culture on inulin, with the best growth for $F$. prausnitzii co-culture with B. catenulatum $\left(\mathrm{OD}_{650}=0.79 \pm 0.02\right)$. 
For B. catenulatum KCTC 3221 and B. animalis ATCC 25527, the overall growth of the co-culture was better than that of Bifidobacterium and F. prausnitzii alone. However, in the glucose-containing medium, B. bifidum BGN4 and B. longum RD72 did not show noticeable changes in growth between monoculture and co-culture. We also observed some strain-specific differences in the growth of B. animalis species (Figure S1). Collectively, these observations highlight the strain- and species-dependent differences in the growth of bifidobacteria.

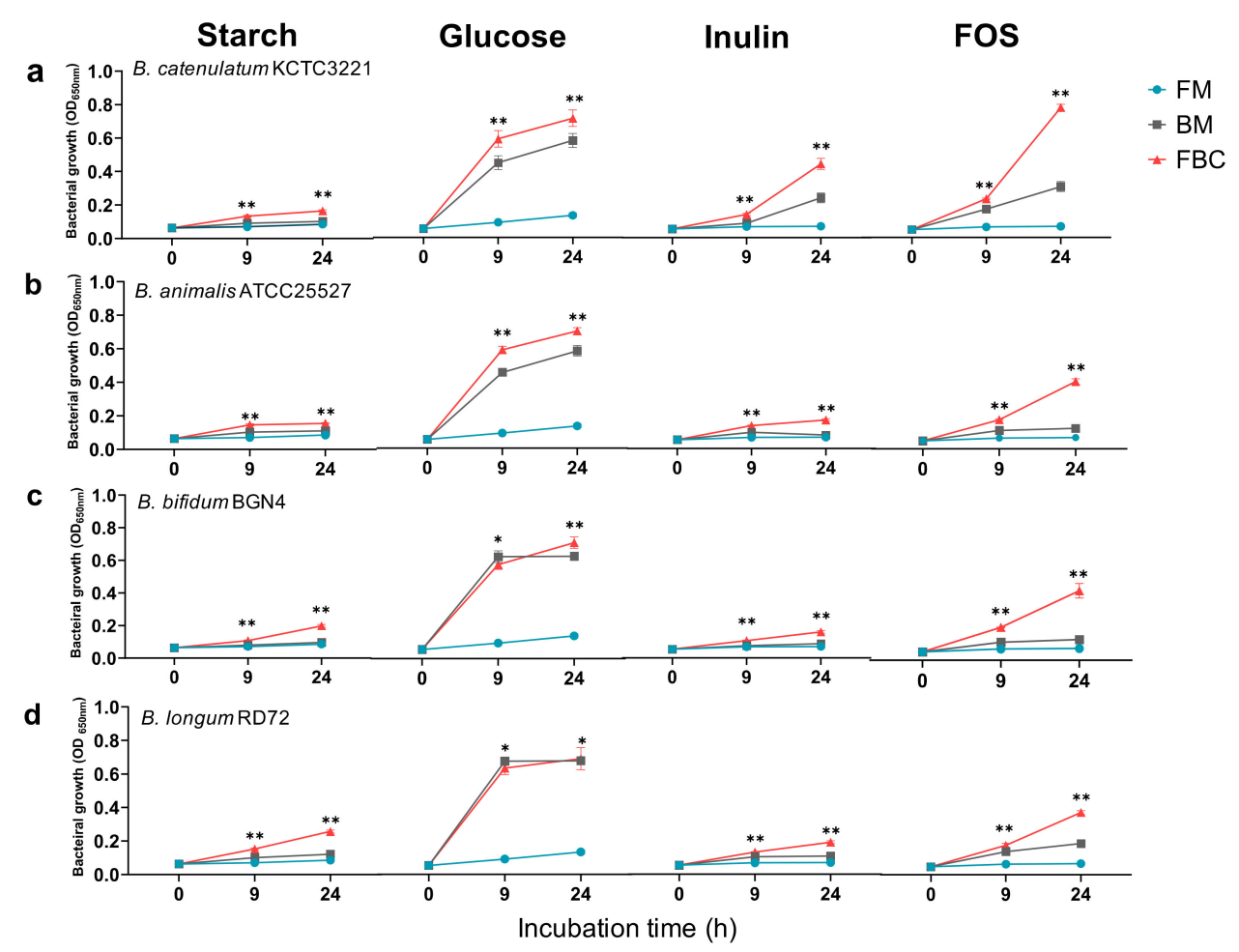

Figure 1. Growth of F. prausnitzii ATCC 27768 with different Bifidobacterium species in liquid culture in the presence of various carbohydrates. Culture $\mathrm{OD}_{650}$ was determined at various time points after inoculation. F. prausnitzii growth in monoculture and co-culture with B. catenulatum KCTC 3221 (a), B. animalis ATCC 25527 (b), B. bifidum BGN4 (c), and B. longum RD72 (d) is shown. All growth experiments were performed in the YCFA medium without SCFA supplemented with starch, glucose, inulin, or FOS, for $24 \mathrm{~h}$. The data are presented as the mean \pm standard deviation $(n=4)$. Statistically significant differences vs. FM group are denoted $\left({ }^{*} p<0.05,{ }^{* *} p<0.01\right)$. FM, monoculture of F. prausnitzii; BM, monoculture of Bifidobacterium; FBC, co-culture of F. prausnitzii and Bifidobacterium.

3.1.2. SCFA Production by Bacteria in Mono- and Co-Culture in the Presence of Different Carbon Sources

We next determined the SCFA levels in different mono- and co-cultures after 9 and $24 \mathrm{~h}$ of cultivation (Figure 2). Only a small amount of SCFA was produced in the starch-containing medium, reflecting the weak growth of bacteria in this medium. Most SCFA were produced in the glucose-containing medium, which also reflected the highest bacterial growth in this medium. The main SCFA produced on glucose was acetate. Further, more acetate was present in co-culture after $24 \mathrm{~h}$ than after $9 \mathrm{~h}$, regardless of the Bifidobacterium species used. B. catenulatum KCTC 3221, B. bifidum BGN4, and B. longum RD72 produced lactate. The bacteria produced less lactate in co-culture than in monoculture. B. catenulatum KCTC 3221 and B. bifidum BGN4 produced more butyrate than B. animalis ATCC 27768 and B. longum RD72 $(4.61 \pm 1.50 \mathrm{mM}$ and $4.85 \pm 0.37 \mathrm{mM}$ vs. $1.85 \pm 0.69 \mathrm{mM}$ and $2.44 \pm 0.96 \mathrm{mM}$, respectively). In the inulin-containing medium, the bacteria produced most acetate in monoculture, while butyrate was the major SCFA after $24 \mathrm{~h}$ of co-culture. We noted similar trends in the FOS-containing medium, although this medium generally supported higher SCFA production than the inulin-containing 
medium. The main SCFA produced on FOS was butyrate after $24 \mathrm{~h}$ of co-culture with F. prausnitzii $(11.17 \pm 0.05 \mathrm{mM}, 4.99 \pm 0.71 \mathrm{mM}, 3.69 \pm 0.56 \mathrm{mM}$, and $6.19 \pm 1.26 \mathrm{mM}$, respectively). Further, butyrate concentrations were significantly higher in every Bifidobacteria co-cultures than in the corresponding F. prausnitzii monocultures. In addition, unlike on glucose, acetate levels after $24 \mathrm{~h}$ of co-culture were lower than those after $9 \mathrm{~h}$ of co-culture. We observed the greatest increase in butyrate levels between 9 and $24 \mathrm{~h}$ incubation in F. prausnitzii ATCC 27768 co-culture with B. catenulatum KCTC 3221 in the presence of FOS $(1.24 \pm 1.40 \mathrm{mM}$ vs. $11.17 \pm 0.05 \mathrm{mM}$, respectively). Further, different $B$. animalis strains produced different amounts of SCFA (Figure S2). B. animalis subsp. lactis RD68 and B. animalis subsp. lactis $\mathrm{SH} 5$ produced more butyrate in the glucose-containing medium than in the FOS-containing medium after $24 \mathrm{~h}$ of co-culture with F. prausnitzii $(6.56 \mathrm{mM}, 8.66 \mathrm{mM}, 6.12 \mathrm{mM}$, and $5.51 \mathrm{mM}$ in respective B. animalis strains). Finally, B. animalis subsp. lactis Ad011 produced large amounts of lactate on all carbon sources tested (Figure S2). Taken together, these observations indicate that the effect of co-culture on SCFA production was species-/strain-specific and dependent on the type of carbon sources.

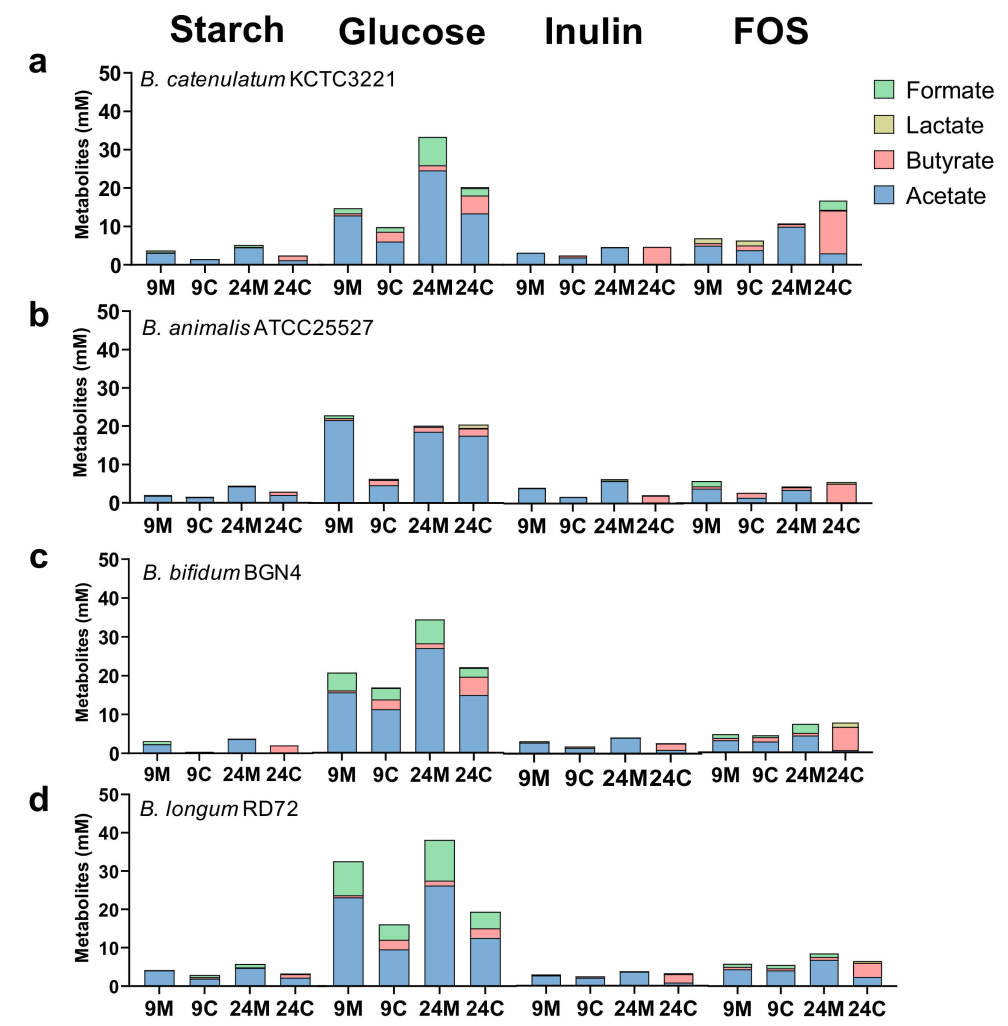

Figure 2. Production of SCFA by bacteria in monoculture and co-culture. Concentrations of SCFA (acetate, lactate, formate, and butyrate) were determined 9 and $24 \mathrm{~h}$ after inoculation. Data for F. prausnitzii in monoculture and in co-culture with B. catenulatum KCTC 3221 (a), B. animalis ATCC 25527 (b), B. bifidum BGN4 (c), and B. longum RD72 (d) are shown. All experiments were performed in YCFA medium without SCFA supplemented with starch, glucose, inulin, or FOS. The data are presented as the mean $(n=3) .9 \mathrm{M}$, metabolites from F. prausnitzii and Bifidobacterium monocultures after $9 \mathrm{~h}$; 9C, metabolites in F. prausnitzii and Bifidobacterium co-culture after $9 \mathrm{~h} ; 24 \mathrm{M}$, metabolites from F. prausnitzii and Bifidobacterium monocultures after $24 \mathrm{~h} ; 24 \mathrm{C}$, metabolites in F. prausnitzii and Bifidobacterium co-culture after $24 \mathrm{~h}$.

3.1.3. Effect of F. prausnitzii ATCC 27768 Co-Culture with B. catenulatum KCTC 3221 or B. animalis ATCC 25527 in a Medium Containing FOS on Culture $\mathrm{pH}$ and Viable Cell Counts

B. catenulatum KCTC 3221 and B. animalis ATCC 25527 showed distinct growth patterns in the FOS-containing media. We followed up these observations by viable cell-count and $\mathrm{pH}$ analyses in 
the FOS medium (Figure 3). The number of bacteria in F. prausnitzii monoculture decreased after $24 \mathrm{~h}$ (6.82 to $6.45 \mathrm{CFU} / \mathrm{mL}$ ) (Figure 3a,c). By contrast, in co-culture with Bifidobacterium, the number of F. prausnitzii cells increased over time. In the case of B. catenulatum KCTC 3221, both the Bifidobacterium and F. prausnitzii grew well in co-culture. Further, F. prausnitzii in co-culture grew more rapidly between 18 and $24 \mathrm{~h}$ than during the initial $9 \mathrm{~h}$ ( $\log \mathrm{CFU} / \mathrm{mL}$ change of 7.64 to 8.13 vs. 6.66 to 6.87 , respectively). Interestingly, although the culture $\mathrm{pH}$ dropped substantially in the F. prausnitzii co-culture with B. catenulatum KCTC 3221 (pH 7.34 to 5.28) (Figure 3b), F. prausnitzii grew well under these conditions. In co-culture with B. animalis ATCC 25527, although the Bifidobacterium did not grow well, it stimulated the growth of F. prausnitzii. B. animalis subsp. lactis RD68 showed a similar growth-promoting effect on F. prausnitzii (Figure S3). The culture $\mathrm{pH}$ in F. prausnitzii co-culture with B. animalis subsp. lactis RD68 did not decrease below 6.0, and this represented the low growth of RD68 strain in the FOS-containing medium. These data suggested that the increased viable cell number of F. prausnitzii in the F. prausnitzii and B. catenulatum co-culture in the FOS-containing medium was related to the highest amount of butyrate produced by F. prausnitzii, and B. catenulatum facilitated it with rapid digestion of dietary fiber and acetate production.
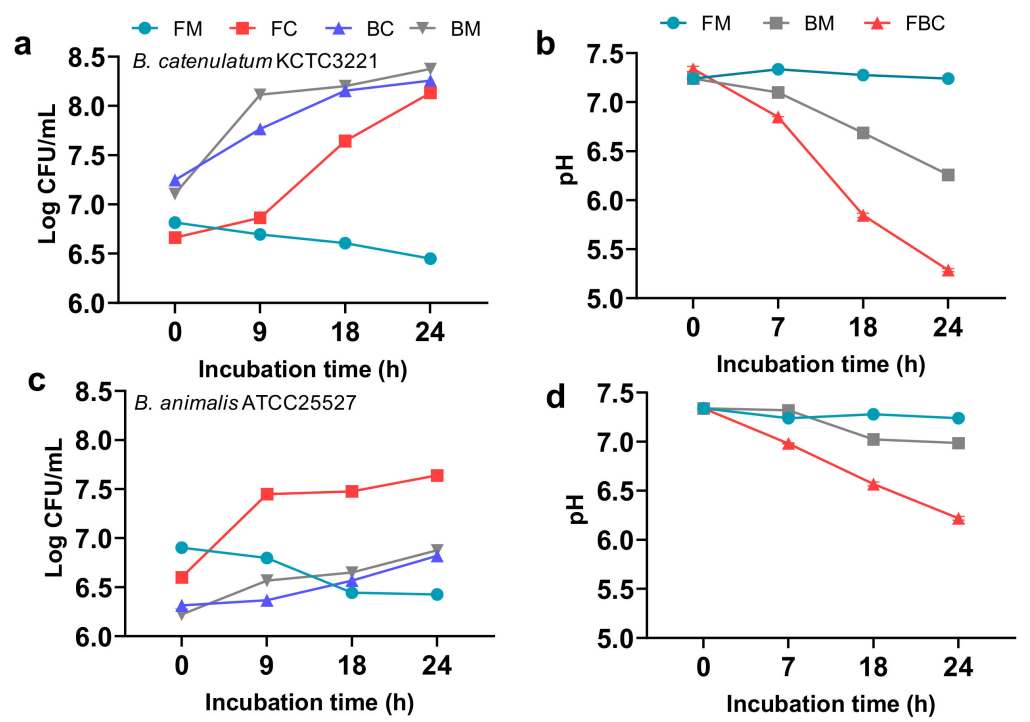

Figure 3. Changes in the viable cell number and $\mathrm{pH}$ of cultures of F. prausnitzii and B. catenulatum KCTC 3221 or B. animalis ATCC 25527 in YCFOS medium. Data for F. prausnitzii in monoculture or co-culture with B. catenulatum KCTC $3221(\mathbf{a}, \mathbf{b})$ or B. animalis ATCC 25527 (c,d) in YC medium supplemented with FOS are shown. Samples were analyzed at the indicated time intervals. Cell numbers were determined by viable counts, as CFU/mL, after plating on LYBHI agar $(\mathbf{a}, \mathbf{c})$. The $\mathrm{pH}$ changes are plotted in $(\mathbf{b}, \mathbf{d})$. The data are presented as the mean \pm standard deviation $(n=3)$. FM, F. prausnitzii in monoculture; BM, Bifidobacterium in monoculture; FC, F. prausnitzii in co-culture; BC, Bifidobacterium in co-culture;FBC, co-culture of F. prausnitzii and Bifidobacterium.

3.2. Comparison of the Fecal Microbiota of Normal Mice Fed F. prausnitzii ATCC 27768 and B. catenulatum KCTC 3221

We next investigated whether B. catenulatum increased the survival of $F$. prausnitzii in a normal mouse gut. We fed the mice bacteria for 2 weeks and then extracted bacterial genomic DNA from the feces and performed 16S rRNA amplicon sequencing and RT-qPCR analysis (Figure 4a). As shown in Figure 4, the intake of bacteria affected the composition of total fecal microbiota in mouse. Further, the number of Faecalibacterium in the feces of animals fed F. prausnitzii and B. catenulatum was significantly higher than that in the feces of animals fed F. prausnitzii. To investigate changes in bacterial richness and homogeneity in mouse feces, we calculated the Shannon and evenness indices (the alpha-diversity indices). The Shannon (Figure 4b) and evenness indices (Figure 4c) in mice fed Faecalibacterium alone (F group) were significantly higher than those in mice fed Bifidobacterium 
alone (B group) $(p<0.05)$. However, the alpha-diversity between the F group and the FB group was not significantly different. We used the Bray-Curtis distance analysis to calculate the beta-diversity of samples in order to determine microbial phylogenetic similarity among the groups (Figure 4d). The structure of the microbial community in the control ( $n$ ) group was similar to that in the F group, and the composition of the microbial community in the mice FB group) was similar to that in the B group.

a

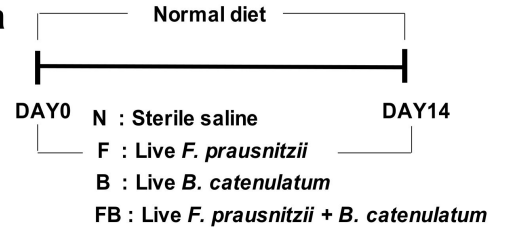

d

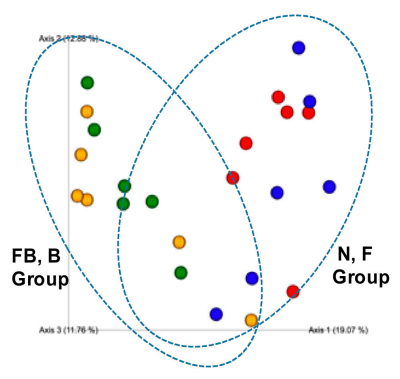

$\mathbf{f}$

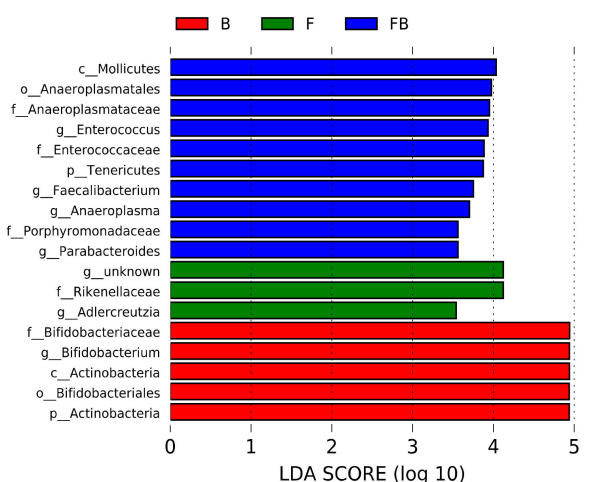

b

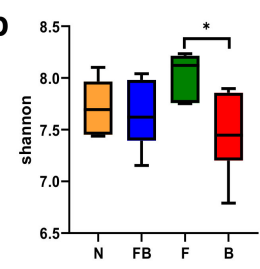

e

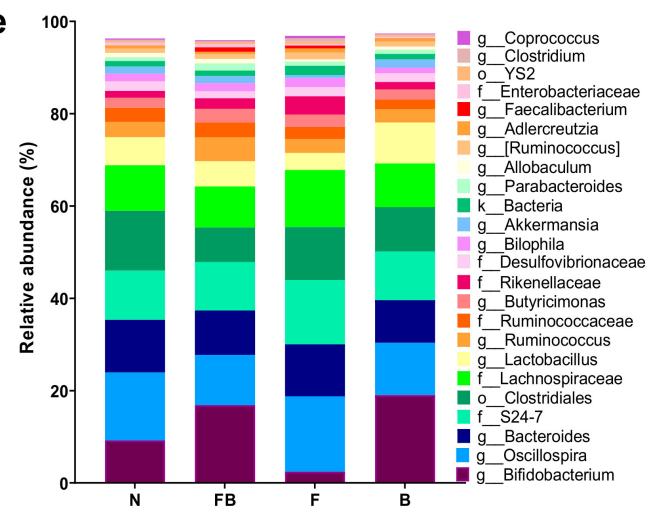

g

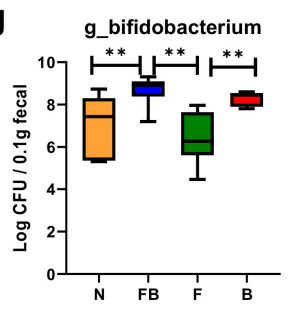

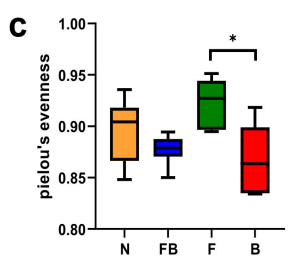

h

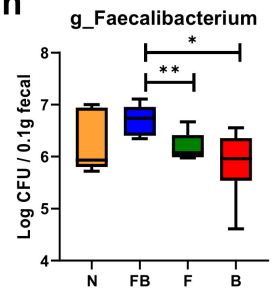

Figure 4. Fecal microbial composition in normal C57B6 mice fed F. prausnitzii and B. catenulatum. The microbiome was analyzed in mouse stool samples (6 mice per group). The mice were administered live bacteria or saline for $14 \mathrm{~d}$ (a). The alpha-diversity indices of microbial community richness and evenness are shown in (b) and (c). The beta-diversity based on the Bray-Curtis distance is shown in (d). The average relative abundance at the genus level (taxa with relative abundance $>0.5 \%$ ) is shown in (e). Significantly different taxa in the B (red), F (green), and FB (blue) groups, determined by LEfSe analysis (threshold $>2.0$ ), are shown in (f). The abundance of F. prausnitzii (g) and Bifidobacterium (h) in the feces was detected by RT-qPCR. Statistically significant differences are shown $\left.{ }^{*} p<0.05,{ }^{* *} p<0.01\right)$. N, normal mouse; FB, mice administered F. prausnitzii $\left(1 \times 10^{9} \mathrm{CFU} / \mathrm{mL}\right)$ and B. catenulatum $\left(1 \times 10^{9} \mathrm{CFU} / \mathrm{mL}\right) ; \mathrm{F}$, mice administered $\mathrm{F}$. prausnitzii $\left(1 \times 10^{9} \mathrm{CFU} / \mathrm{mL}\right) ; \mathrm{B}$, mice administered B. catenulatum $\left(1 \times 10^{9} \mathrm{CFU} / \mathrm{mL}\right)$.

Taxonomic analysis of the microbial composition at the genus level revealed different microbial composition in the four groups (Figure 4e). We also used the LEfSe analysis to identify bacteria specific for the treatment groups (Figure 4f). In the analysis, an LDA score greater than 2.0 reflects species with a significantly different abundance between the groups. Faecalibacterium was highly abundant in the FB microbiome, while Actinobacteria and Bifidobacteriales were enriched in the B group. The microbial communities in the $n$ and FB groups were strikingly different. Finally, we determined the abundances of Faecalibacterium and Bifidobacterium genera in the feces by RT-qPCR (Figure 4g,h). Faecalibacterium 
was significantly more abundant in the FB group feces than in the F group feces $(p<0.01)$ (Figure $4 \mathrm{~g}$ ). Further, the feces from the FB and B groups contained significantly more bifidobacteria than those from the $n$ and F groups ( $p<0.01$ ) (Figure 4 h). However, bifidobacterial abundances in the FB and B group feces were not significantly different.

\subsection{Effect of Bacterial Culture Supernatant on Proinflammatory Cytokine Production by HT-29 Cells and RAW} 264.7 Cells, and in the Colon in the DSS-Induced Colitis Mouse Model

F. prausnitzii has anti-inflammatory effects in vitro and in vivo [4-7]. We first investigated the anti-inflammatory effects of FB (co-culture) supernatant against LPS-induced inflammation in vitro. To do this, we assayed inflammatory cytokine levels in HT-29 cells and RAW 264.7 cells exposed to different culture supernatants. As shown in Figure 5a, HT-29 cells treated with FB supernatant produced significantly less IL-8 than the negative control group $(p<0.001)$. IL-8 release was significantly lower upon exposure to the FB supernatant than that after exposure to either F or B supernatant (from monocultures). In addition, the effect of FB supernatant was similar to that of $1 \mathrm{mM}$ sodium butyrate. Further, exposure to FB supernatant significantly blocked the release of LPS-induced cytokines IL-6 and TNF- $\alpha$ by RAW 264.7 macrophages ( $p<0.0001$; Figure $5 b, c)$. As above, the effect of the FB supernatant was similar to that of $1 \mathrm{mM}$ sodium butyrate.

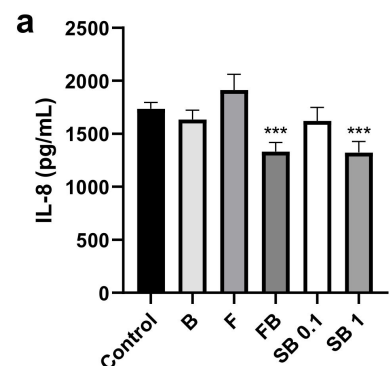

d

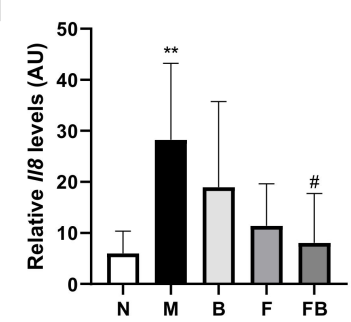

b

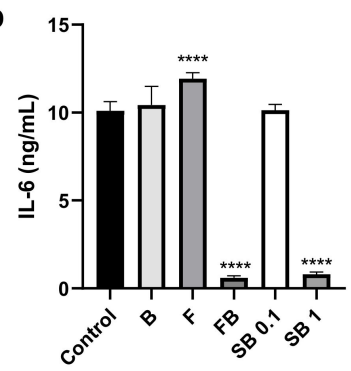

e

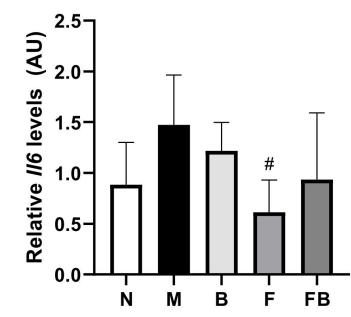

C

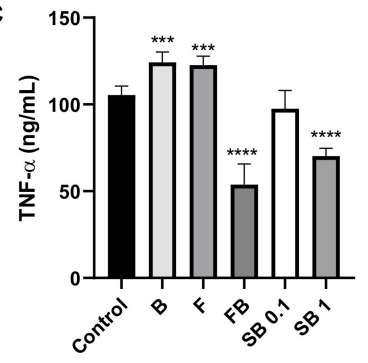

f

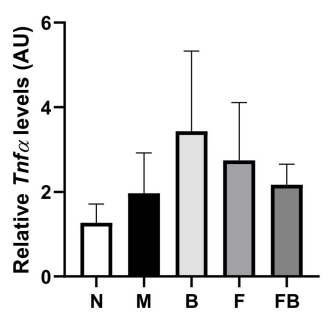

Figure 5. Immunomodulatory effects of bacterial culture supernatants in vitro $(\mathbf{a}-\mathbf{c})$ and in vivo (d-f). $(\mathbf{a}-\mathbf{c})$ In vitro effect of the culture supernatants: effect on the secretion of IL-8 by LPS-stimulated HT-29 cells (a), and on the secretion of IL-6 (b) and TNF- $\alpha$ (c) by LPS-stimulated RAW 264.7 macrophages. In $\mathbf{a}-\mathbf{c}$, the cells were exposed to sodium butyrate as the positive control. The cells were stimulated with $100 \mathrm{ng} / \mathrm{mL}$ LPS prior to the experiment, and cytokine levels were determined by using commercial enzyme-linked immunosorbent assay kits. (d-f) In vivo effect of the culture supernatants: relative mRNA levels determined by RT-qPCR for the genes of proinflammatory cytokines, Il8, Il6, and Tnf- $\alpha$, in the colon of DSS-induced colitis mice. All data are shown as the mean + standard deviation. $(\mathbf{a}-\mathbf{c})$ Control, $10 \%$ YCFOS medium; B, 10\% supernatant of B. catenulatum culture; F, $10 \%$ supernatant of $F$. prausnitzii culture; FB, $10 \%$ supernatant of F. prausnitzii and B. catenulatum co-culture medium; SB 0.1, $0.1 \mathrm{mM}$ sodium butyrate; SB1, $1 \mathrm{mM}$ sodium butyrate. Significant differences compared with the control group, ${ }^{* * *} p<0.001,{ }^{* * * *} p<0.0001(n=3)$. (d-f) N, untreated mice; M, DSS-induced colitis mice administered with culture medium control; B, DSS-induced colitis mice administered with medium from B. catenulatum culture; F, DSS-induced colitis mice administered with medium from F. prausnitzii culture; FB, DSS-induced colitis mice administered with medium from F. prausnitzii and B. catenulatum co-culture. Significant differences compared with the N group, ${ }^{* *} p<0.01$; compared with the M group, $\# p<0.05(n=6)$. 
We also determined the effect of bacterial supernatant on the production of proinflammatory cytokines in vivo. Specifically, we used RT-qPCR to investigate changes in the expression of genes encoding IL-8, IL-6, and TNF- $\alpha$ cytokines in the colon of DSS-treated mice fed different bacterial culture supernatants. DSS treatment increased the Il $(p<0.01)$ and Il6 mRNA levels (M vs. $n$ group in Figure $5 \mathrm{~d}$,e). In contrast, feeding FB supernatant significantly reduced the $I l 8 \mathrm{mRNA}$ levels compared with the control group treated with DSS (M group) $(p<0.05)$ (Figure $5 d$ ). Feeding F supernatant significantly reduced the Il6 mRNA levels in the colon compared with control $\mathrm{M}$ group $(p<0.05)$. Feeding M, B, F, and FB supernatants did not significantly affect Tnf- $\alpha$ mRNA levels.

\subsection{Effect of Co-Culture Bacterial Supernatant on DSS-Induced Colitis in Mouse}

DSS-induced colitis is a commonly used model in IBD research. To evaluate the anti-inflammatory effect of the FB bacterial culture supernatant on DSS-induced colitis, we established DSS-induced colitis in mice (Figure 6a). The mice were fed ad libitum 2\% (w/v) DSS in drinking water for $7 \mathrm{~d}$, followed by normal tap water for $2 \mathrm{~d}$; they were concomitantly fed the bacterial supernatant. At the end of the experiment, we determined the levels of four major SCFAs (acetate, propionate, lactate, and butyrate) in the cecum (Figure $6 \mathrm{~b}$ ). The cecum of mice fed the FB supernatant contained significantly more butyrate than the cecum of the $n$ and M groups $(p<0.05)$. Further, the cecum of mice in the FB group contained significantly more acetate and propionate than the cecum in the M group. The Bray-Curtis distance analysis revealed distinct differences in the beta-diversity between the $\mathrm{M}, n$, and FB groups (Figure 6c). We also used LEfSe to identify the dominant colonic microbiota in the M and FB groups. Pairwise comparison revealed an increased abundance of Akkermensia and Verrucomicrobiales in the FB group compared with the M group (Figure 6d). Further, feeding FB supernatant significantly increased the relative abundance of Akkermansia compared with the $n$ group $(p<0.05$; Figure 6e). Finally, as determined by H\&E staining, feeding $2 \%$ DSS resulted in widely distributed intestinal epithelial damage, inflammatory cell infiltration, and edema in the colon (Figure 6f). In contrast, the intestinal epithelium in the FB group was relatively intact. 
a

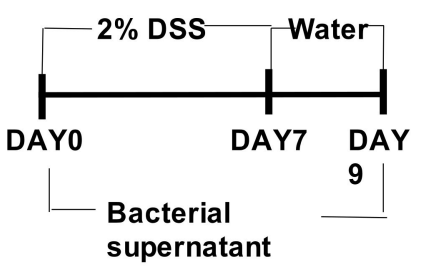

b

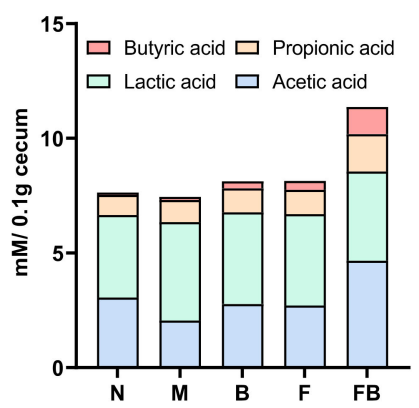

C

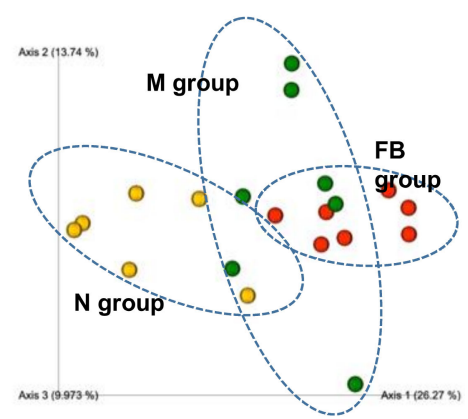

e

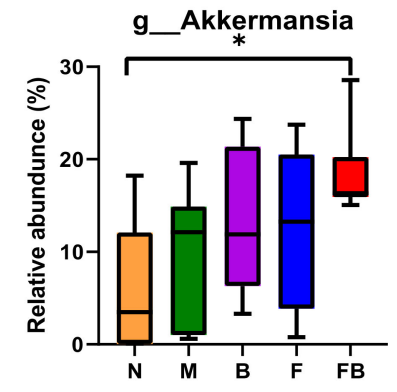

$2 \%$ DSS + B medium
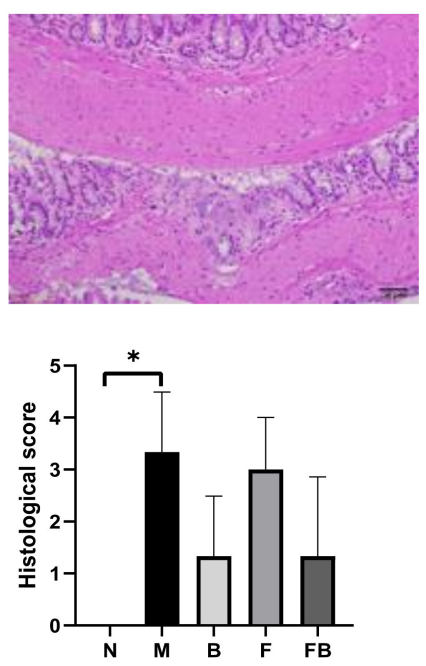

Figure 6. Effect of supernatant from bacterial co-culture on DSS-induced colitis in a mouse model. Colitis was induced in mice by feeding $2 \%(w / v)$ DSS for $7 \mathrm{~d}$; the mice were concomitantly administered bacterial supernatant (a). The SCFA levels and microbiome were analyzed in mouse cecum samples (6 mice per group). The levels of butyrate, propionate, lactate, and acetate in the cecum are shown in (b). The beta-diversity based on the Bray-Curtis distance is shown in (c). Significantly different taxa in the FB (red) and M (green) groups were identified by LEfSe analysis (threshold >2.0), as shown in (d). Relative abundance of the genus Akkermansia is shown in (e). Histopathological changes in the colon are presented in (f). Representative H\&E-stained colon sections are shown for each group, $\times 50$ magnification, with the histological scores. The data are shown as the mean \pm standard deviation $(n=6)$. Statistically significant differences vs. untreated mice $\left({ }^{*} p<0.05\right)$. N, untreated mice; M, DSS-induced colitis mice administered culture medium control; FB, DSS-induced colitis mice administered with medium from F. prausnitzii and B. catenulatum co-culture; F, DSS-induced colitis mice administered with medium from F. prausnitzii culture; B, DSS-induced colitis mice administered with medium from B. catenulatum culture. 


\section{Discussion}

Breakdown of microbial homeostasis can lead to IBD. F. prausnitzii is a good biomarker for a healthy gut, and its abundance is reduced in Crohn's disease [14]. The bacterium induces the production of anti-inflammatory factors, such as IL-10, and produces butyrate. In the current study, we aimed to determine the factors that improve the growth and mammalian gut colonization of F. prausnitzii. We screened several bifidobacteria strains which have a nutritional cross-feeding relationship with $F$. prausnitzii to increase the viability of $F$. prausnitzii in the gut. We also investigated the anti-inflammatory effect of metabolites produced in F. prausnitzii co-culture with Bifidobacterium on intestinal inflammation in a DSS-induced colitis model.

IBD is a chronic inflammatory disease characterized by such clinical symptoms as weight loss, diarrhea, and rectal bleeding. In the current study, we induced IBD in mouse by administering DSS in drinking water. This IBD model is one of the best-characterized animal models that mimic human IBD symptoms. According to recent studies, live F. prausnitzii cells or the bacterial culture supernatant alleviate inflammation in DSS-induced colitis. However, because of the stringent growth conditions of F. prausnitzii, an extremely oxygen-sensitive bacterium, intestine delivery of viable cells is one of the current challenges precluding its use as a probiotic [8]. Indeed, we confirmed here that administration of $F$. prausnitzii culture supernatant from co-culture significantly increased the overall viability and colonization efficiency of $F$. prausnitzii and alleviated the colitis symptoms in mouse compared to culture supernatant from monoculture.

Bacteria in the large intestine compete for nutrients; however, metabolic byproducts secreted by one strain can be an essential energy source for another [37]. F. prausnitzii is reported to utilize the fermentation end-products of a few colonic bacteria including Bacteroides thetaiotaomicron and E. coli [26]. Bifidobacterial ability to utilize carbon sources is strain-specific, and these bacteria use various non-digestible carbohydrates, such as resistant starch, inulin, and FOS. Bifidobacteria that use a monosaccharides decomposition pathway as a main carbohydrate catabolism compete with F. prausnitzii for survival and growth [38]. However, Bifidobacterium strains capable of metabolizing non-digestible carbon sources, e.g., B. catenulatum, could support the carbohydrate availability of acetate-dependent butyrate producers such as F. prausnitzii by producing a high amount of acetate as an end-product in a nutrient-restricted environment [39]. In the present study, bifidobacteria, alone or in co-culture with $F$. prausnitzii, grew most rapidly during the initial $9 \mathrm{~h}$ of culture in a medium containing glucose, and during 9 to $24 \mathrm{~h}$ of incubation in a medium containing FOS. This suggests that the ability of bifidobacteria to degrade long-chain carbohydrates may affect the growth of other bacteria in co-culture. Based on the viable cell counts during growth on FOS, we showed that B. catenulatum KCTC 3221 grew similarly well in co-culture and monoculture. F. prausnitzii did not grow well during the first $9 \mathrm{~h}$ in co-culture with B. catenulatum KCTC 3221 but showed a 29-fold growth increase during the ensuing 9-24 h. It is feasible that the co-culture promoted the growth of F. prausnitzii by lowering the medium $\mathrm{pH}$ (to between 5.8 and 6.5) and supplying glycolysis metabolites, in addition to supplying acetate, as a result of carbon source degradation by Bifidobacterium. Improved butyrate production by F. prausnitzii in co-culture with B. adolescentis in the FOS-containing medium was reported previously [25]. In that study, bacterial growth was only determined by OD measurements, and it was hard to assess the growth-promoting ability of the strain. In order to improve the data interpretation, we followed the growth of each bacterium in co-culture by using viable cell counts as well. Further, we showed here that among the bifidobacterial strains tested, B. catenulatum KCTC 3221 most notably enhanced growth and butyrate production by F. prausnitzii. B. catenulatum is the most frequently detected species in adults and is present in $38 \%$ of individuals [40] and effective against acute liver damage [41,42]. Besides anti-inflammatory effects, butyrate has benefits to regulate pathological progress in metabolic diseases, including non-alcoholic fatty liver diseases and liver fibrosis. Further research investigating the effects of co-cultured B. catenulatum and F. prausnitzii on other types of inflammatory and metabolic diseases is required to evaluate the applicability as a potential candidate. 
The above effects were strain-specific rather than characteristic for the entire Bifidobacterium genus or certain species. We here compared the bacterial growth rate $\left(\mathrm{OD}_{650}\right)$ and SCFA levels in the cultures of four B. animalis strains. B. animalis subsp. lactis Ad011 grew rapidly during the first $9 \mathrm{~h}$ and produced copious amounts of lactate in monoculture and co-culture in most media tested. In a medium containing glucose, while B. animalis ATCC 25527 grew better than B. animalis subsp. lactis RD68 alone, the growth of both strains was similar in co-culture. However, the SCFA levels were different in co-cultures of these strains: B. animalis ATCC 25527 produced similar amounts of acetate and butyrate after 9 and $24 \mathrm{~h}$, but $B$. animalis subsp. lactis RD68 produced less acetate and more butyrate after $24 \mathrm{~h}$ than after $9 \mathrm{~h}$. This suggests that while moderate growth in co-culture on glucose of two different bacterial species has a synergistic effect on their growth, excessive growth of one bacterium can interfere with the growth of the other bacterium. In the FOS-containing medium, the co-cultures of B. animalis ATCC 25527 and B. animalis subsp. lactis RD68 with F. prausnitzii grew more rapidly in the period between 9 and $24 \mathrm{~h}$ than during the initial $9 \mathrm{~h}$. On the other hand, the co-culture with $B$. animalis subsp. lactis SH5 showed similar growth in the two time periods. The co-culture with B. animalis subsp. lactis ad011 grew during the initial $9 \mathrm{~h}$ and then stopped. This emphasizes the notion that different strains of the same species show different growth characteristics on the same sugar source, not only in the monoculture, but also in co-culture with $F$. prausnitzii.

It is well-established that F. prausnitzii consumes acetate produced by Bifidobacteria and produces butyrate $[24,25]$. In the current study, we confirmed a decrease in acetate levels and an increase in butyrate levels after $24 \mathrm{~h}$ of culture, compared with those after $9 \mathrm{~h}$. F. prausnitzii requires a carbohydrate energy source for growth and butyrate formation, and bifidobacteria do not produce butyrate. The observed changes in acetate and butyrate levels in co-cultures in the YCFOS medium not supplemented with SCFA suggest that the growth of F. prausnitzii was supported by acetate supplied by Bifidobacterium, while the two bacteria competed for FOS present in the culture medium. In contrast, during co-culture in the glucose- and starch-containing media, the acetate levels in the medium were higher after $24 \mathrm{~h}$ than those after $9 \mathrm{~h}$. Carbon source utilization allowed for a rapid Bifidobacterium growth, with an increased production of acetate and the slowing of growth of $F$. prausnitzii, without a notable increase in butyrate levels. Hence, the interaction between the two bacteria depends on the type of carbon source in co-culture. Others have similarly reported increased butyrate levels and reduced acetate levels in F. prausnitzii co-culture with Bifidobacterium in FOS- or inulin-containing media $[25,39,43]$. FOS is a major source of carbon that can be used as an energy source by bacteria in the large intestine. It is not degraded in the upper digestive system, prior to passage to the large intestine [21]. In the current study, we confirmed that the bacteria grew better in a medium containing FOS than in a medium containing inulin, a longer-chain compound than FOS. F. prausnitzii and B. catenulatum produced the most butyrate in the FOS-containing medium.

To evaluate the intestinal delivery of F. prausnitzii, we orally administered the bacteria to healthy mice. In another study, live cultures of $F$. prausnitzii were fed to newborn calves to observe the effects on animal health and fecal microorganisms [42]. The group fed F. prausnitzii showed reduced rates of diarrhea and mortality. Further, fecal microbial analysis revealed that the relative abundance of F. prausnitzii and Firmicutes in feces increased 3 to 5 weeks since the start of bacterial feeding [44]. In the current study, based on the RT-qPCR analysis and fecal microbiome analysis, we showed that Faecalibacterium was significantly more abundant in the FB and F mice than in untreated mice. Bifidobacterium abundance was significantly higher in the FB and B groups than in untreated mice, while the relative abundance of Faecalibacterium was higher in the FB mice than in the F mice. Faecalibacterium does not easily colonize the intestine. However, ingestion of Faecalibacterium together with Bifidobacterium appeared to improve its intestinal colonization. As evidenced by the in vitro experiments described above, Bifidobacterium influences the growth of Faecalibacterium, which may explain the in vivo effects.

In the current study, we also evaluated the anti-inflammatory effect of FB bacterial supernatant in the colon of DSS-induced colitic mouse and on LPS-stimulated HT-29 cells and RAW 264.7 macrophages. 
The colonic Il8 mRNA levels in the FB group were significantly lower than those in the DSS-induced control group. This was similar to the trend observed in the experiments with LPS-induced HT-29 colon cells. Further, FB culture supernatant inhibited TNF- $\alpha$ and IL- 6 secretion by LPS-stimulated RAW 264.7 macrophages. LPS activates the innate immune system and induces the release of cytokines (TNF- $\alpha$ and IL-6) and chemokines (IL-8) [45]. According to previous studies, F. prausnitzii monoculture supernatant reduces NF-KB activation and IL-8 secretion in vitro [7-9,46]. However, the effect of supernatants of F. prausnitzii co-culture with Bifidobacterium on IL-8 levels has not been evaluated prior to the current study. The FB supernatant used for cell treatment in this study contained approximately 0.8-1.2 mM butyrate. Accordingly, the extent of cytokine level reduction elicited by the FB supernatant was similar to the effect of $1 \mathrm{mM}$ sodium butyrate. Sodium butyrate inhibits the phosphorylation of AKT and the NF-кB P65 signaling pathway, with anti-inflammatory activity in mice treated with 2,4,6-trinitrobenzene sulfonic acid [15,47].

The effect of the Faecalibacterium supernatant on DSS-induced colitis in mice has been reported previously $[48,49]$. However, the ability of the supernatant to relieve inflammation in animals is controversial [11]. In the current study, the amount of butyrate in the cecum was significantly higher in the FB group than in other groups. This could be related to the amount of butyrate in the FB supernatant. The mechanism underpinning the effect of the FB supernatant could be explained by the reported beneficial effect of sodium butyrate on the abundance of Akkermansia muciniphila. Butyrate consumption decreases the $\mathrm{pH}$ in the colon, which stimulates the abundance of some bacteria, e.g., Eubacterium cylindroides. These bacteria promote mucin secretion within the mucus layer, which increases the abundance of $A$. muciniphila [50,51]. Akkermensia degrades the mucus, leading to a release of oligosaccharides and the production of propionate and acetate [52-54]. Analysis of the cecal microflora in the current study suggests that the relative abundance of Akkermansia in the FB group was significantly higher than that in the control group. A. muciniphila regulates the expression of genes involved in the host immunity, metabolism, and gut barrier functions. Both A. muciniphila and F. prausnitzii are present in the intestinal mucosa and are considered members of healthy gut microflora. The abundance of both species is reduced in several intestinal disorders (e.g., IBD). Therefore, the co-treatment of both live F. prausnitzii and B. catenulatum bacteria and supernatants can modulate the gut microbial community and alleviate the pathological progress of related diseases.

Although we here demonstrated the effectiveness of bifidobacteria in improving the intestinal delivery of Faecalibacterium, the study has some limitations. We showed that the growth of F. prausnitzii and bifidobacteria depends on the type of dietary fiber in vitro. Ingestion of FOS, a prebiotic, could further improve the delivery of Faecalibacterium to the intestine when the two bacteria are ingested together in vivo. However, because the composition of the large intestine of the mouse and human is different, further experiments are needed to determine whether colonization of F. prausnitzii in human would be similarly improved.

Taken together, we have shown here that the administration of F. prausnitzii together with bifidobacteria improves the intestinal delivery of the former. In addition, we showed that the co-culture of $F$. prausnitzii with bifidobacteria results in an increased production of butyrate, an immunomodulatory compound. Further, the supernatant from Faecalibacterium co-culture with bifidobacteria inhibited the production of proinflammatory cytokines in vitro and in vivo. Changes in the intestinal microflora upon supernatant administration were confirmed by $16 S$ rRNA metagenomic analysis. Bifiodbacterium genus includes several probiotic strains, while F. prausnitzii is a good next-generation probiotic candidate. Administration of F. prausnitzii together with bifidobacteria shows great potential not only for maintaining intestinal homeostasis, but also as a treatment for IBD.

Supplementary Materials: The following are available online at http://www.mdpi.com/2076-2607/8/5/788/s1: Figure S1. Growth of F. prausnitzii ATCC 27768 with different Bifidobacterium animalis strains in liquid culture in the presence of various carbohydrates. Figure S2. Production of SCFA by bacteria in monoculture and co-culture. Concentrations of SCFA (acetate, lactate, formate, and butyrate) were determined 9 and $24 \mathrm{~h}$ after inoculation. Figure S3. Changes in the viable cell number and $\mathrm{pH}$ of cultures of F. prausnitzii and B. animalis subsp. lactis RD68 in YCFOS medium. 
Author Contributions: Conceptualization, H.K., H.J.Y. and G.E.J.; methodology, H.K., S.K. and H.J.Y.; formal analysis, H.K. and H.J.Y.; data curation, Y.J. and H.J.Y.; writing-Original draft preparation, H.K.; writing-Review and editing, H.J.Y. and G.E.J.; funding acquisition, G.E.J. All authors have read and agreed to the published version of the manuscript.

Funding: This research was supported by the Bio \& Medical Technology Development Program of the National Research Foundation (NRF) and funded by the Korean government (MSIT) (NRF-2017M3A9F3041747).

Conflicts of Interest: The authors declare no conflict of interest.

\section{References}

1. Kaplan, G.G.; Ng, S.C. Understanding and preventing the global increase of inflammatory bowel disease. Gastroenterology 2017, 152, 313-321. [CrossRef] [PubMed]

2. M'koma, A.E. Inflammatory bowel disease: An expanding global health problem. Clinical Medicine Insights: Gastroenterology 2013, 6, 33-47. [CrossRef] [PubMed]

3. Pithadia, A.B.; Jain, S. Treatment of inflammatory bowel disease (IBD). Pharmacol. Rep. 2011, 63, 629-642. [CrossRef]

4. Rosen, M.J.; Dhawan, A.; Saeed, S.A. Inflammatory bowel disease in children and adolescents. JAMA Pediatr. 2015, 169, 1053-1060. [CrossRef] [PubMed]

5. Khan, I.; Ullah, N.; Zha, L.; Bai, Y.; Khan, A.; Zhao, T.; Che, T.; Zhang, C. Alteration of Gut Microbiota in Inflammatory Bowel Disease (IBD): Cause or Consequence? IBD Treatment Targeting the Gut Microbiome. Pathogens 2019, 8, 126. [CrossRef]

6. Fakhoury, M.; Negrulj, R.; Mooranian, A.; Al-Salami, H. Inflammatory bowel disease: Clinical aspects and treatments. J. Inflamm. Res. 2014, 7, 113. [CrossRef]

7. Martín, R.; Miquel, S.; Benevides, L.; Bridonneau, C.; Robert, V.; Hudault, S.; Chain, F.; Berteau, O.; Azevedo, V.; Chatel, J.M. Functional characterization of novel Faecalibacterium prausnitzii strains isolated from healthy volunteers: A step forward in the use of F. prausnitzii as a next-generation probiotic. Front. Microbiol. 2017, $8,1226$.

8. Martín, R.; Bermúdez-Humarán, L.G.; Langella, P. Searching for the bacterial effector: The example of the multi-skilled commensal bacterium Faecalibacterium prausnitzii. Front. Microbiol. 2018, 9, 346.

9. Sokol, H.; Pigneur, B.; Watterlot, L.; Lakhdari, O.; Bermúdez-Humarán, L.G.; Gratadoux, J.-J.; Blugeon, S.; Bridonneau, C.; Furet, J.-P.; Corthier, G. Faecalibacterium prausnitzii is an anti-inflammatory commensal bacterium identified by gut microbiota analysis of Crohn disease patients. Proc. Natl. Acad. Sci. USA 2008, 105, 16731-16736. [CrossRef]

10. Carlsson, A.H.; Yakymenko, O.; Olivier, I.; Håkansson, F.; Postma, E.; Keita, Å.V.; Söderholm, J.D. Faecalibacterium prausnitzii supernatant improves intestinal barrier function in mice DSS colitis. Scand. J. Gastroenterol. 2013, 48, 1136-1144. [CrossRef]

11. Kawade, Y.; Sakai, M.; Okamori, M.; Morita, M.; Mizushima, K.; Ueda, T.; Takagi, T.; Naito, Y.; Itoh, Y.; Shimada, T. Administration of live, but not inactivated, Faecalibacterium prausnitzii has a preventive effect on dextran sodium sulfate-induced colitis in mice. Mol. Med. Rep. 2019, 20, 25-32. [CrossRef] [PubMed]

12. Martín, R.; Miquel, S.; Chain, F.; Natividad, J.M.; Jury, J.; Lu, J.; Sokol, H.; Theodorou, V.; Bercik, P.; Verdu, E.F. Faecalibacterium prausnitzii prevents physiological damages in a chronic low-grade inflammation murine model. BMC Microbiol. 2015, 15, 67. [CrossRef] [PubMed]

13. Rossi, O.; Khan, M.T.; Schwarzer, M.; Hudcovic, T.; Srutkova, D.; Duncan, S.H.; Stolte, E.H.; Kozakova, H.; Flint, H.J.; Samsom, J.N. Faecalibacterium prausnitzii strain HTF-F and its extracellular polymeric matrix attenuate clinical parameters in DSS-induced colitis. PLoS ONE 2015, 10, e0123013. [CrossRef] [PubMed]

14. Lopez-Siles, M.; Duncan, S.H.; Garcia-Gil, L.J.; Martinez-Medina, M. Faecalibacterium prausnitzii: From microbiology to diagnostics and prognostics. ISME J. 2017, 11, 841-852. [CrossRef] [PubMed]

15. Venegas, D.P.; Marjorie, K.; Landskron, G.; González, M.J.; Quera, R.; Dijkstra, G.; Harmsen, H.J.; Faber, K.N.; Hermoso, M.A. Short chain fatty acids (SCFAs)-mediated gut epithelial and immune regulation and its relevance for inflammatory bowel diseases. Front. Immunol. 2019, 10.

16. Zhang, M.; Zhou, L.; Wang, Y.; Dorfman, R.G.; Tang, D.; Xu, L.; Pan, Y.; Zhou, Q.; Li, Y.; Yin, Y. Faecalibacterium prausnitzii produces butyrate to decrease c-myc-related metabolism and th17 differentiation by inhibiting histone deacetylase 3. Int. Immunol. 2019, 31, 499-514. [CrossRef] 
17. Zhou, L.; Zhang, M.; Wang, Y.; Dorfman, R.G.; Liu, H.; Yu, T.; Chen, X.; Tang, D.; Xu, L.; Yin, Y. Faecalibacterium prausnitzii produces butyrate to maintain Th17/Treg balance and to ameliorate colorectal colitis by inhibiting histone deacetylase 1. Inflamm. Bowel Dis. 2018, 24, 1926-1940. [CrossRef]

18. Hamer, H.M.; Jonkers, D.; Venema, K.; Vanhoutvin, S.; Troost, F.; Brummer, R.J. The role of butyrate on colonic function. Aliment. Pharm. Ther. 2008, 27, 104-119. [CrossRef]

19. Macfarlane, G.T.; Gibson, G.R. Carbohydrate fermentation, energy transduction and gas metabolism in the human large intestine. In Gastrointestinal Microbiology; Springer: Boston, MA, USA; pp. 269-318.

20. Wolin, M.J.; Miller, T.L.; Yerry, S.; Zhang, Y.; Bank, S.; Weaver, G.A. Changes of fermentation pathways of fecal microbial communities associated with a drug treatment that increases dietary starch in the human colon. Appl. Environ. Microbiol. 1999, 65, 2807-2812. [CrossRef]

21. Flint, H.J.; Scott, K.P.; Duncan, S.H.; Louis, P.; Forano, E. Microbial degradation of complex carbohydrates in the gut. Gut Microbes 2012, 3, 289-306. [CrossRef]

22. Duncan, S.H.; Holtrop, G.; Lobley, G.E.; Calder, A.G.; Stewart, C.S.; Flint, H.J. Contribution of acetate to butyrate formation by human faecal bacteria. Br. J. Nutr. 2004, 91, 915-923. [CrossRef] [PubMed]

23. Nagpal, R.; Kurakawa, T.; Tsuji, H.; Takahashi, T.; Kawashima, K.; Nagata, S.; Nomoto, K.; Yamashiro, Y. Evolution of gut Bifidobacterium population in healthy Japanese infants over the first three years of life: A quantitative assessment. Sci. Rep. 2017, 7, 1-11. [CrossRef] [PubMed]

24. Duncan, S.H.; Barcenilla, A.; Stewart, C.S.; Pryde, S.E.; Flint, H.J. Acetate utilization and butyryl coenzyme A (CoA): Acetate-CoA transferase in butyrate-producing bacteria from the human large intestine. Appl. Environ. Microbiol. 2002, 68, 5186-5190. [CrossRef] [PubMed]

25. Rios-Covian, D.; Gueimonde, M.; Duncan, S.H.; Flint, H.J.; de los Reyes-Gavilan, C.G. Enhanced butyrate formation by cross-feeding between Faecalibacterium prausnitzii and Bifidobacterium adolescentis. FEMS Microbiol. Lett. 2015, 362. [CrossRef] [PubMed]

26. Foditsch, C.; Santos, T.M.; Teixeira, A.G.; Pereira, R.V.; Dias, J.M.; Gaeta, N.; Bicalho, R.C. Isolation and characterization of Faecalibacterium prausnitzii from calves and piglets. PLoS ONE 2014, 9, e116465. [CrossRef] [PubMed]

27. Khan, M.T.; Duncan, S.H.; Stams, A.J.; van Dijl, J.M.; Flint, H.J.; Harmsen, H.J. The gut anaerobe Faecalibacterium prausnitzii uses an extracellular electron shuttle to grow at oxic-anoxic interphases. ISME J. 2012, 6, 1578-1585. [CrossRef]

28. Miquel, S.; Martín, R.; Lashermes, A.; Gillet, M.; Meleine, M.; Gelot, A.; Eschalier, A.; Ardid, D.; Bermúdez-Humarán, L.; Sokol, H. Anti-nociceptive effect of Faecalibacterium prausnitzii in non-inflammatory IBS-like models. Sci. Rep. 2016, 6, 19399. [CrossRef]

29. Kang, S.; You, H.J.; Lee, Y.-G.; Jeong, Y.; Johnston, T.V.; Baek, N.-I.; Ku, S.; Ji, G.E. Production, Structural Characterization, and In Vitro Assessment of the Prebiotic Potential of Butyl-Fructooligosaccharides. Int. J. Mol. Sci. 2020, 21, 445. [CrossRef]

30. Brown, K.; Abbott, D.W.; Uwiera, R.R.; Inglis, G.D. Removal of the cecum affects intestinal fermentation, enteric bacterial community structure, and acute colitis in mice. Gut Microbes 2018, 9, 218-235. [CrossRef]

31. Kang, S.; Denman, S.E.; Morrison, M.; Yu, Z.; Dore, J.; Leclerc, M.; McSweeney, C.S. Dysbiosis of fecal microbiota in Crohn's disease patients as revealed by a custom phylogenetic microarray. Inflamm. Bowel Dis. 2010, 16, 2034-2042. [CrossRef]

32. Fijan, S.; Šulc, D.; Steyer, A. Study of the in vitro antagonistic activity of various single-strain and multi-strain probiotics against Escherichia coli. Int. J. Env. Res. Public Health 2018, 15, 1539. [CrossRef] [PubMed]

33. Kim, J.-K.; Lee, S.H.; Lee, S.-Y.; Kim, E.-K.; Kwon, J.-E.; Seo, H.-B.; Lee, H.H.; Lee, B.-I.; Park, S.-H.; Cho, M.-L. Grim19 attenuates DSS induced colitis in an animal model. PLoS ONE 2016, 11, e0155853. [CrossRef] [PubMed]

34. Fachi, J.L.; Felipe, J.D.; Pral, L.P.; da Silva, B.K.; Corrêa, R.O.; de Andrade, M.C.P.; da Fonseca, D.M.; Basso, P.J.; Câmara, N.O.S.; Souza, É.L.D.S.E. Butyrate protects mice from Clostridium difficile-induced colitis through an HIF-1-dependent mechanism. Cell Rep. 2019, 27, 750-761. [CrossRef] [PubMed]

35. Lee, H.J.; Oh, S.-H.; Jang, H.W.; Kwon, J.-H.; Lee, K.J.; Kim, C.H.; Park, S.J.; Hong, S.P.; Cheon, J.H.; Kim, T.I. Long-term effects of bone marrow-derived mesenchymal stem cells in dextran sulfate sodium-induced murine chronic colitis. Gut Liver 2016, 10, 412. [CrossRef] 
36. Bhatt, B.; Zeng, P.; Zhu, H.; Sivaprakasam, S.; Li, S.; Xiao, H.; Dong, L.; Shiao, P.; Kolhe, R.; Patel, N. Gpr109a limits microbiota-induced IL-23 production to constrain ILC3-mediated colonic inflammation. J. Immunol. 2018, 200, 2905-2914. [CrossRef]

37. Smith, N.W.; Shorten, P.R.; Altermann, E.; Roy, N.C.; McNabb, W.C. The classification and evolution of bacterial cross-feeding. Front. Ecol. Evol. 2019, 7, 153. [CrossRef]

38. Rivière, A.; Selak, M.; Lantin, D.; Leroy, F.; de Vuyst, L. Bifidobacteria and butyrate-producing colon bacteria: Importance and strategies for their stimulation in the human gut. Front. Microbiol. 2016, 7, 979. [CrossRef]

39. Moens, F.; Rivière, A.; Selak, M.; de Vuyst, L. Inulin-type fructan degradation capacity of interesting butyrate-producing colon bacteria and cross-feeding interactions of Faecalibacterium prausnitzii DSM 17677 T with bifidobacteria. Arch. Public Health 2014, 72, O6. [CrossRef]

40. Matsuki, T.; Watanabe, K.; Tanaka, R.; Fukuda, M.; Oyaizu, H. Distribution of bifidobacterial species in human intestinal microflora examined with 16S rRNA-gene-targeted species-specific primers. Appl. Environ.Microbol. 1999, 65, 4506-4512. [CrossRef]

41. Fang, D.; Shi, D.; Lv, L.; Gu, S.; Wu, W.; Chen, Y.; Guo, J.; Li, A.; Hu, X.; Guo, F. Bifidobacterium pseudocatenulatum LI09 and Bifidobacterium catenulatum LI10 attenuate D-galactosamine-induced liver injury by modifying the gut microbiota. Sci. Rep. 2017, 7, 1-13. [CrossRef]

42. Xing, H.C.; Li, L.J.; Xu, K.J.; Shen, T.; Chen, Y.B.; Sheng, J.F.; Chen, Y.; Fu, S.Z.; Chen, C.L.; Wang, J.G. Protective role of supplement with foreign Bifidobacterium and Lactobacillus in experimental hepatic ischemia-reperfusion injury. J. Gastroenterol. Hepatol. 2006, 21, 647-656. [CrossRef] [PubMed]

43. Scott, K.P.; Martin, J.C.; Duncan, S.H.; Flint, H.J. Prebiotic stimulation of human colonic butyrate-producing bacteria and bifidobacteria, In Vitro. FEMS Microbiol. Lett. 2014, 87, 30-40. [CrossRef] [PubMed]

44. Foditsch, C.; Pereira, R.V.V.; Ganda, E.K.; Gomez, M.S.; Marques, E.C.; Santin, T.; Bicalho, R.C. Oral administration of Faecalibacterium prausnitzii decreased the incidence of severe diarrhea and related mortality rate and increased weight gain in preweaned dairy heifers. PLoS ONE 2015, 10, e0145485. [CrossRef] [PubMed]

45. Chen, B.-C.; Liao, C.-C.; Hsu, M.-J.; Liao, Y.-T.; Lin, C.-C.; Sheu, J.-R.; Lin, C.-H. Peptidoglycan-induced IL-6 production in RAW 264.7 macrophages is mediated by cyclooxygenase-2, PGE2/PGE4 receptors, protein kinase A, IкB kinase, and NF-кB. J. Immunol. 2006, 177, 681-693. [CrossRef]

46. Breyner, N.M.; Michon, C.; de Sousa, C.S.; Boas, P.B.V.; Chain, F.; Azevedo, V.A.; Langella, P.; Chatel, J.M. Microbial anti-inflammatory molecule (MAM) from Faecalibacterium prausnitzii shows a protective effect on DNBS and DSS-induced colitis model in mice through inhibition of NF-kB pathway. Front. Microbiol. 2017, 8, 114. [CrossRef]

47. Chen, G.; Ran, X.; Li, B.; Li, Y.; He, D.; Huang, B.; Fu, S.; Liu, J.; Wang, W. Sodium butyrate inhibits inflammation and maintains epithelium barrier integrity in a TNBS-induced inflammatory bowel disease mice model. EBioMedicine 2018, 30, 317-325. [CrossRef]

48. Huang, X.-L.; Zhang, X.; Fei, X.-Y.; Chen, Z.-G.; Hao, Y.-P.; Zhang, S.; Zhang, M.-M.; Yu, Y.-Q.; Yu, C.-G. Faecalibacterium prausnitzii supernatant ameliorates dextran sulfate sodium induced colitis by regulating Th17 cell differentiation. World J. Gastroenterol. 2016, 22, 5201. [CrossRef]

49. Zhang, M.; Qiu, X.; Zhang, H.; Yang, X.; Hong, N.; Yang, Y.; Chen, H.; Yu, C. Faecalibacterium prausnitzii inhibits interleukin-17 to ameliorate colorectal colitis in rats. PLOS ONE 2014, 9, e109146. [CrossRef]

50. Alrafas, H.R.D.; Busbee, B.P.; Nagarkatti, P.; Nagarkatti, M. Effect of Sodium Butyrate Supplementation on the Gut Microbiome during Colorectal Cancer. J. Immunol. 2019, 202 (Suppl. 1), 191.10.

51. Roshanravan, N.; Mahdavi, R.; Jafarabadi, M.A.; Alizadeh, E.; Ghavami, A.; Saadat, Y.R.; Alamdari, N.M.; Dastouri, M.R.; Alipour, S.; Ostadrahimi, A. The effects of sodium butyrate and high-performance inulin supplementation on the promotion of gut bacterium Akkermansia muciniphila growth and alterations in miR-375 and KLF5 expression in type 2 diabetic patients: A randomized, double-blind, placebo-controlled trial. Eur. J. Integr. Med. 2018, 18, 1-7.

52. Lopez-Siles, M.; Enrich-Capó, N.; Aldeguer, X.; Sabat-Mir, M.; Duncan, S.H.; Garcia-Gil, L.J.; Martinez-Medina, M. Alterations in the abundance and co-occurrence of Akkermansia muciniphila and Faecalibacterium prausnitzii in the colonic mucosa of inflammatory bowel disease subjects. Front. Cell. Infect. Microbiol. 2018, 8, 281. [CrossRef] [PubMed] 
53. Lukovac, S.; Belzer, C.; Pellis, L.; Keijser, B.J.; de Vos, W.M.; Montijn, R.C.; Roeselers, G. Differential modulation by Akkermansia muciniphila and Faecalibacterium prausnitzii of host peripheral lipid metabolism and histone acetylation in mouse gut organoids. MBio 2014, 5, e01438-14. [CrossRef] [PubMed]

54. Zhang, Z.; Wu, X.; Cao, S.; Cromie, M.; Shen, Y.; Feng, Y.; Yang, H.; Li, L. Chlorogenic acid ameliorates experimental colitis by promoting growth of Akkermansia in mice. Nutrients 2017, 9, 677. [CrossRef] [PubMed]

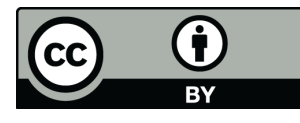

(C) 2020 by the authors. Licensee MDPI, Basel, Switzerland. This article is an open access article distributed under the terms and conditions of the Creative Commons Attribution (CC BY) license (http://creativecommons.org/licenses/by/4.0/). 